Radiation dose enhancement at tissue-tungsten interfaces in HDR brachytherapy

This content has been downloaded from IOPscience. Please scroll down to see the full text. 2014 Phys. Med. Biol. 596659

(http://iopscience.iop.org/0031-9155/59/21/6659)

View the table of contents for this issue, or go to the journal homepage for more

Download details:

IP Address: 138.25.78.25

This content was downloaded on 23/03/2015 at 05:11

Please note that terms and conditions apply. 


\title{
Radiation dose enhancement at tissue- tungsten interfaces in HDR brachytherapy
}

\author{
Z Han ${ }^{1}$, M Safavi-Naeini ${ }^{1}$, S Alnaghy ${ }^{1}$, DL Cutajar ${ }^{1}$, \\ S Guatelli ${ }^{1}$, M Petasecca ${ }^{1}$, DR Franklin ${ }^{2}$, A Malaroda ${ }^{1}$, \\ M Carrara ${ }^{3}$, J Bucci ${ }^{4}$, M Zaider ${ }^{5}$, MLF Lerch ${ }^{1}$ and \\ AB Rosenfeld
}

${ }^{1}$ Centre for Medical Radiation Physics, University of Wollongong, Wollongong, NSW 2522, Australia

2 Faculty of Engineering and IT, UTS, Ultimo, NSW 2007, Australia

3 Medical Physics Unit, Dept. of Diagnostic Imaging and Radiotherapy, Fondazione IRCCS Istituto Nazionale dei Tumori, Milan, Italy

${ }^{4}$ St George Hospital Cancer Care Centre, St George, NSW 2540, Australia

5 Memorial Sloan Kettering Cancer Center, New York, NY 10021, USA

E-mail: anatoly@uow.edu.au

Received 19 July 2014, revised 3 September 2014

Accepted for publication 10 September 2014

Published 17 October 2014

\begin{abstract}
HDR BrachyView is a novel in-body dosimetric imaging system for real-time monitoring and verification of the source position in high dose rate (HDR) prostate brachytherapy treatment. It is based on a high-resolution pixelated detector array with a semi-cylindrical multi-pinhole tungsten collimator and is designed to fit inside a compact rectal probe, and is able to resolve the 3D position of the source with a maximum error of $1.5 \mathrm{~mm}$. This paper presents an evaluation of the additional dose that will be delivered to the patient as a result of backscatter radiation from the collimator. Monte Carlo simulations of planar and cylindrical collimators embedded in a tissue-equivalent phantom were performed using Geant 4 , with an ${ }^{192}$ Ir source placed at two different sourcecollimator distances. The planar configuration was replicated experimentally to validate the simulations, with a MOSkin dosimetry probe used to measure dose at three distances from the collimator. For the cylindrical collimator simulation, backscatter dose enhancement was calculated as a function of axial and azimuthal displacement, and dose distribution maps were generated at three distances from the collimator surface. Although significant backscatter dose enhancement was observed for both geometries immediately adjacent to the collimator, simulations and experiments indicate that backscatter dose is negligible at distances beyond $1 \mathrm{~mm}$ from the collimator. Since HDR BrachyView is enclosed within a $1 \mathrm{~mm}$ thick tissue-equivalent plastic shell, all
\end{abstract}


backscatter radiation resulting from its use will therefore be absorbed before reaching the rectal wall or other tissues. dosimetry, brachytherapy, HDR

Keywords: backscatter dose, BSDF, in vivo dosimetry, brachytherapy, HDR

(Some figures may appear in colour only in the online journal)

\section{Introduction}

Successful high dose rate prostate brachytherapy treatment (HDR PBT) relies on accurate dose delivery and is therefore strongly dependent on the accurate placement of the highly radioactive source within the prostate volume (Valentin 2005, Williamson 2006, Rivard et al 2009). Tracking and monitoring of the source throughout the treatment procedure is necessary to minimise post-operative complications resulting from source misplacement (Thomadsen et al 2003, Cunningham et al 2010, Batic et al 2011, Kertzscher et al 2011).

An advanced real-time transrectal source tracking probe, BrachyView, is currently under development by the Centre for Medical Radiation Physics (CMRP) at the University of Wollongong. The probe consists of an imaging plane (a $15 \times 60 \mathrm{~mm}$ pixellated silicon detector) encased within a half-cylindrical multi-pinhole tungsten collimator. It is designed to resolve the three-dimensional location of the source inside the treatment volume to an accuracy of $1.5 \mathrm{~mm}$ with a sub-second temporal resolution (Safavi-Naeini et al 2013). Tungsten was selected as the collimator material due to its high density and high atomic mass, which result in good collimation despite the volumetric constraints of the probe. However, the presence of a high- $Z$ inhomogeneity in the irradiated patient results in attenuation of the radiation through the inhomogeneity and causes local perturbations known as interface effects (Reft et al 2003). The interaction of primary and scattered photons emitted by the source incident on the collimator surface produce an enhancement in the dose delivered immediately adjacent to the collimator surface (Regulla et al 1998, Nadrowitz et al 2001, Beaulieu et al 2012). This effect is mainly due to the generation of backscattered secondary electrons (i.e. photo- and Auger electrons) in the most superficial layers of the high- $Z$ material (Das and Chopra 1995). Depending on the range of these secondary electrons, it is possible that tissue damage to the anterior wall of the rectum could result due to its proximity to the collimator. Crucially, dose calculation algorithms used by the clinical treatment planning systems based on the AAPM TG-43 (Nath et al 1995) and the more recent TG-186 protocols (Beaulieu et al 2012) cannot accurately calculate the dose near a high- $Z$ inhomogeneity. Only Monte Carlo simulations can estimate the dose in this region with sufficient precision to determine whether the backscatter dose is likely to be hazardous to nearby tissues; therefore dedicated simulations and/or experimental measurements are required. To date, little work has been done to evaluate the dose enhancement due to backscatter, particularly from tungsten inserts and for the range of photon energies used in HDR PBT, since tungsten and tungsten alloys are rarely used for permanent medical implants.

Substantial work exists on the measurement and effects of backscatter radiation in specific medical applications, primarily related to the consequences of the irradiation of permanent metallic implants used in dental prostheses and bone repairs. Radiation mucositis has been observed in tissue immediately adjacent to regions with high electron densities (such as gold crowns, amalgam fillings and mandibular reconstructions) in patients treated with therapeutic radiation in the head and neck region (Castillo et al 1988, Regulla et al 1998). Regulla et al have exposed single layers of mouse embryonic fibroblasts in contact with a thin metallic gold foil and enclosed within a tissue equivalent polymethylmethacrylate 
(PMMA) phantom to heavily filtered x-ray beams with mean effective energies of 40 $100 \mathrm{keV}$ (Regulla et al 1998). Radiation dose and cell survival curves were compared to those obtained from irradiation of cells held between two PMMA control plates. An increase of $55 \%$ to $114 \%$ in dose was measured within the $\approx 100 \mu \mathrm{m}$ air gap formed between the gold surface and the detector (a $0.1 \mu \mathrm{m}$ thick Beryllium oxide $(\mathrm{BeO})$ layer deposited on a thermally stimulated exoelectron emission (TSSE) substrate). The biological effect of this dose increase was different due to the higher linear energy transfer (LET) of the backscattered low energy electrons; survival curves of the irradiated cells which were in contact with gold lack the pronounced shoulder observed at lower doses and resemble those of cells irradiated with high LET radiation.

Castillo et al have reported a $17 \%$ increase in dose on the entrance side of a stainless steel mandibular implant for a $6 \mathrm{MV}$ photon beam (Castillo et al 1988). Farahani et al have measured the absorbed dose in a tissue equivalent polymer phantom adjacent to various dental restoration materials including 18 carat gold alloy, a silver-mercury dental amalgam, nickelchromium dental casting alloy and human tooth tissue, using GafChromic films (Farahani et al 1990). Results demonstrate an increase in the absorbed dose of up to $100 \%$ in tissue on the backscatter side of the gold insert and up to $20 \%$ adjacent to tooth tissue when irradiated by a collimated ${ }^{60} \mathrm{Co}$ beam. Niroomand-Rad et al have reported an increase in dose of $22.5 \%$ and $20 \%$ for $6 \mathrm{MV}$ and $18 \mathrm{MV}$ photon beams, respectively, at tissue-titanium dental interface in head and neck cancer patients (Niroomand et al 1996). Ravikumar et al have measured the change in the backscatter dose as a function of the photon energy and the width and thickness of the inhomogeneity by placing aluminium, steel, cadmium and lead implants of varying thickness within a tissue equivalent phantom, irradiated by ${ }^{60} \mathrm{Co}$ gamma rays and $6 \mathrm{MV}$ and $18 \mathrm{MV}$ photon beams (Ravikumar et al 2004). The measured backscatter dose was greater with lower energy photons $\left(1.17 \mathrm{MeV}\right.$ and $1.33 \mathrm{MeV}$ photons emitted by ${ }^{60} \mathrm{Co}$ ) for all metallic implants. Chin et al have conducted Monte Carlo simulations studying backscatter effects of various configurations of dental work, demonstrating an increase of up to $33 \%$ in the absorbed dose in patients with metallic dentures undergoing radiotherapy (Chin et al 2009). Their results indicate that the backscatter dose is completely absorbed within the first 3-5 mm of water-equivalent material.

In this paper, the results of experimental and simulation work to study the additional dose deposited by backscatter from the BrachyView transrectal tungsten probe in response to radiation from an ${ }^{192}$ Ir HDR source are presented. Backscatter dose enhancement is quantified using the backscatter dose correction factor (BSDF), first introduced by Das et al., which measures the dose enhancement effect of backscatter resulting from radiation incident on the interface between a low- $Z$ region (such as human tissue) and a high- $Z$ region (such as a metallic surface) (Das and Kahn 1989, Das and Chopra 1995).

An initial simulation of a planar tungsten collimator inside a water-equivalent phantom was conducted, and the BSDF calculated for two source positions as a function of distance $x$ from the interface. The simulation was validated using an identical experimental configuration for several different values of $x$. Finally, a second simulation was performed using identical methodology with an accurate model of the actual cylindrical probe used in the BrachyView design; the BSDF was again calculated at a variety of locations around the collimator.

\section{Materials and methods}

All simulations and experiments described in this section use the BSDF to quantify dose enhancement. The BSDF is defined as the ratio of the dose measured within a homogeneous 


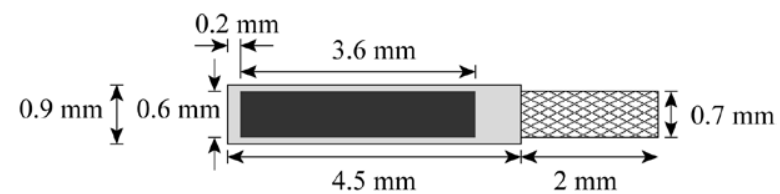

Figure 1. The simulated ${ }^{192}$ Ir HDR brachytherapy source. The core consists of pure iridium with a uniform distribution of ${ }^{192} \mathrm{Ir}$, surrounded by a steel shell (reproduced with permission from Safavi-Naeini et al 2013).

tissue equivalent volume with and without the presence of a high electron density region $\left(D_{i}\right.$ and $D_{h}$, respectively):

$$
\operatorname{BSDF}(E, A, w, d, t, x, Z, \Theta)=\frac{D_{i}}{D_{h}}
$$

where $E$ is the energy of the photon beam, $A$ is cross-sectional area of the field at the point of measurement, $t$ and $w$ are the thickness and the width of the high- $Z$ region, $d$ is the distance between the top surface of the high- $Z$ region and the top surface of the low- $Z$ region, $x$ is the distance between the point of measurement and the top surface of the high- $Z$ region and $\Theta$ is the photon beam angle of incidence (Das and Kahn 1989, Das and Chopra 1995).

Two probe geometries (planar and cylindrical) and an ${ }^{192}$ Ir HDR brachytherapy source were simulated using the Geant 4 toolkit. The source model used throughout the simulations was based on a Nucletron microSelectron HDR ${ }^{192}$ Ir source (also known as the mHDR-v2), as shown in figure 1 (Daskalov et al 1998, Borg and Rogers 1998, Perez-Calatayud et al 2012, Safavi-Naeini et al 2013). The core consists of a pure iridium metal cylinder (3.6 mm long and $0.6 \mathrm{~mm}$ in diameter) with ${ }^{192} \mathrm{Ir}$ uniformly distributed through its core volume, surrounded by a steel shell (4.5 $\mathrm{mm}$ in length with an outer diameter of $0.9 \mathrm{~mm})$, connected to a short steel wire ( $2 \mathrm{~mm}$ long and and $0.7 \mathrm{~mm}$ in diameter). Primary particles were generated using the Geant4 Radioactive Decay Module (G4RadioactiveDecay). The Geant4 Low Energy package (G4LowEnergy) using the Livermore Evaluated Data Libraries (Cullen et al 1997) was employed to model low energy interactions.

\subsection{Planar tungsten collimator}

2.1.1. Monte Carlo simulations. Models of a $90 \times 90 \times 200 \mathrm{~mm}$ water phantom representing soft tissue with and without a $90 \times 50 \times 4 \mathrm{~mm}$ rectangular tungsten plate (representing the HDR BrachyView collimator) placed at the geometric centre were developed in Geant 4 . A $1 \times 1 \times 10 \mathrm{~mm}$ column of water directly above the centre of the top face of the collimator was quantised into one hundred $1 \times 1 \times 0.1 \mathrm{~mm}$ rectangular scoring voxels. One billion primary particles of ${ }^{192} \mathrm{Ir}$ were generated with the source model placed $20 \mathrm{~mm}$ above the centre of the top face of the collimator. A second source position, $43 \mathrm{~mm}$ above the centre of the top face of the collimator, was also simulated with four billion primary particles. The simulation was performed for each source position, firstly with the tungsten collimator inside the water phantom and secondly with the collimator replaced by the same volume of water. The ratio of dose recorded in each scoring voxel with and without the collimator present was calculated to determine the BSDF for the two source positions as a function of distance $x$ from the centre of the front face of the collimator.

Due to the extremely steep dose gradient expected in the immediate proximity of the tungsten collimator, an additional simulation was performed for each source position with $10 \mu \mathrm{m}$ 


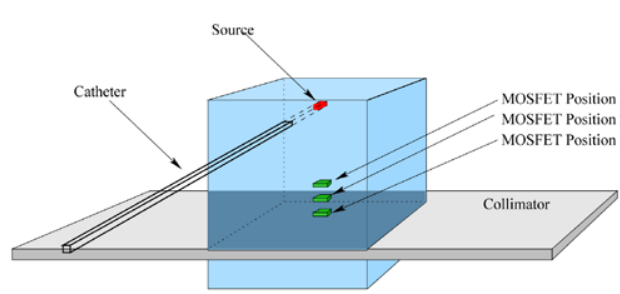

(a)

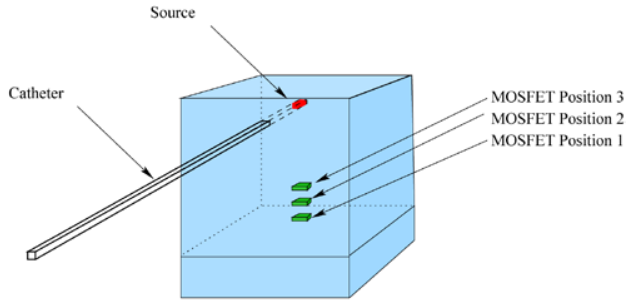

(b)

Figure 2. The experimental configuration; measurements were made at three different points (front face of MOSkin detector placed at $0 \mathrm{~mm}, 5 \mathrm{~mm}$ and $10 \mathrm{~mm}$ above the surface of the tungsten collimator) for two different source positions $(20 \mathrm{~mm}$ and $43 \mathrm{~mm}$ above the surface of the collimator, $43 \mathrm{~mm}$ shown in this figure). The measurements were then repeated after the removal of the collimator. (a) With planar tungsten collimator. $(b)$ Without planar tungsten collimator.

thick rectangular scoring voxels (ten times higher spatial resolution than the previous simulation) covering the region within $1 \mathrm{~mm}$ of the collimator surface.

2.1.2. Experimental configuration. The experiments were conducted at the HDR Brachytherapy facilities at the St. George Hospital Cancer Care Centre, Kogorah. The experimental configuration is illustrated in figure 2. A series of $90 \times 90 \mathrm{~mm}$ Computerized Imaging Reference Systems (CIRS) Plastic Water® LR sheets, each with a thickness of either $3 \mathrm{~mm}$, $5 \mathrm{~mm}$ or $10 \mathrm{~mm}$, were stacked to construct a $90 \times 90 \times 100 \mathrm{~mm}$ tissue equivalent phantom. A $120 \times 50 \times 4 \mathrm{~mm}$ collimator, fabricated from a tungsten alloy $(95 \% \mathrm{~W}, 3.5 \% \mathrm{Ni}$ and $1.5 \% \mathrm{Cu}$ ) was placed immediately below the phantom; in turn, it was supported by a further $90 \times 90 \times 20 \mathrm{~mm}$ slab of Plastic Water. For dose measurements performed in the absence of the tungsten collimator, the tissue-equivalent phantom block was simply placed directly on top of the final Plastic Water slab. One of the $10 \mathrm{~mm}$ sheets in the phantom assembly included a hole for the insertion of a catheter into which the HDR brachytherapy source is inserted by an afterloader mechanism; re-arrangement of the Plastic Water sheets allow the source to be placed either $20 \mathrm{~mm}$ or $43 \mathrm{~mm}$ from the top surface of the collimator.

For each source position, a Nucletron microSelectron HDR afterloader remotely inserted a microSelectron ${ }^{192}$ Ir source into the most distal end of the catheter. Source dwell times were calculated to be equivalent to a $370 \mathrm{GBq}(10 \mathrm{Ci})$ source being positioned for $10 \mathrm{~s}$ and $50 \mathrm{~s}$ at distances of $20 \mathrm{~mm}$ and $43 \mathrm{~mm}$ from the top surface of the tungsten collimator, respectively. Dose was measured at $x=0 \mathrm{~mm}, 5 \mathrm{~mm}$ and $10 \mathrm{~mm}$ from the top surface of the tungsten collimator, using a precalibrated MOSkin ${ }^{\mathrm{TM}}$ detector (Rivard et al 2004). This detector is a MOSFET based detector designed at CMRP with a sensitive volume thickness of $\leqslant 1 \mu \mathrm{m}$, with high reproducibility between devices from the same batch (coefficient of dispersion of $2.5 \%$, measured using an ${ }^{192}$ Ir HDR brachytherapy source) and a linear response up to a maximum threshold voltage of $24 \mathrm{~V}$ (corresponding to an accumulated dose of approximately 70 Gy) (Qi et al 2007, Kwan et al 2008, Kwan et al 2009). For each dose measurement, the detector was placed within a shallow groove, etched on the central axis of the surface of a $90 \times 90 \times 5 \mathrm{~mm}$ Plastic Water sheet and spanning half its length. This allowed the MOSkin detector to be positioned directly beneath the ${ }^{192}$ Ir source at a precise distance from the top surface of the tungsten collimator. The source was remotely moved into position for the desired dwell time by the afterloader, after which it was retracted and safely stowed. 


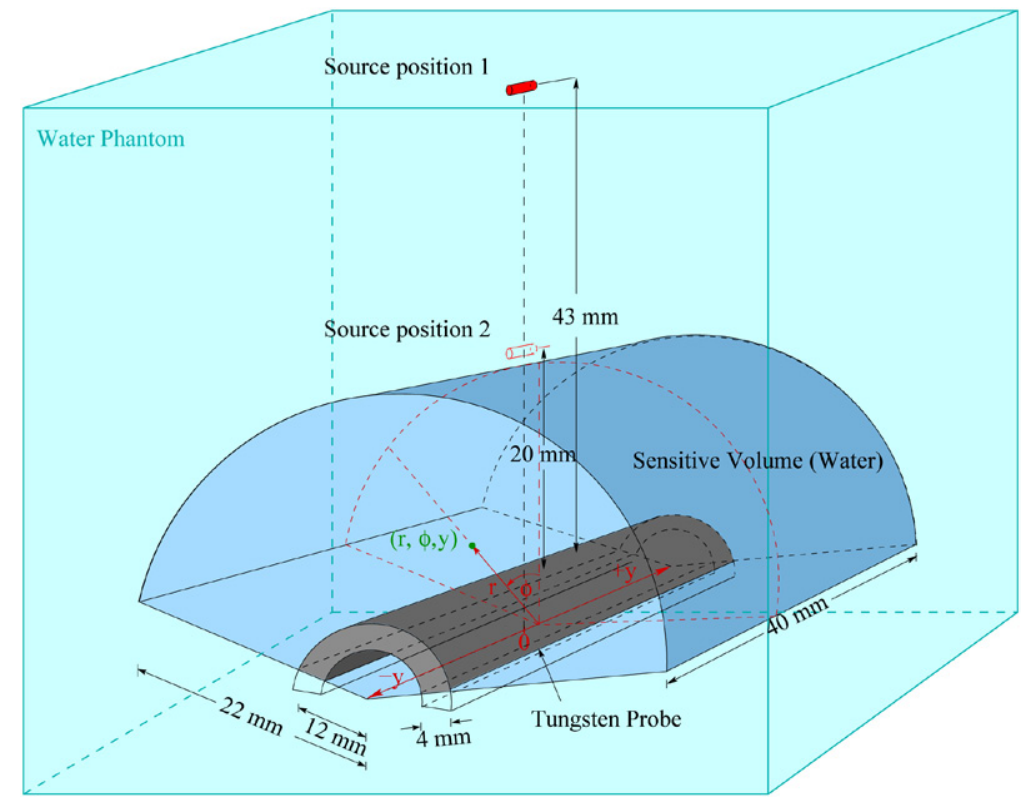

Figure 3. The simulated cylindrical probe, surrounded by the tissue equivalent sensitive volume, which is divided into scoring voxels in a cylindrical coordinate system.

The MOSkin threshold voltage was recorded $30 \mathrm{~s}$ after each irradiation by the CMRP digital MOSFET readout system.

It is anticipated that there will be a very high rate of change in the backscatter dose near the surface of the collimator. Therefore, the actual point inside the MOSkin detector at which the dose is measured needs to be considered. The geometry and composition of the MOSkin detector employed in measuring the dose is such that its effective water-equivalent depth (WED) is approximately $70 \mu \mathrm{m}$ from its front face (Kwan et al 2008). Therefore, when placed in direct contact with the collimator's surface, the dose measurement will actually be performed at a distance of $70 \mu \mathrm{m}$ rather than zero. Similarly, $70 \mu \mathrm{m}$ must be added to $x$ for each additional measurement.

For each source position, the dose measurement procedure was repeated three times at each collimator-sensor distance, with and without the tungsten collimator in place. The change in the threshold voltage was calculated and converted to dose values for each source position. The BSDF was then calculated as the ratio of the calculated dose delivered with and without the tungsten collimator present.

\subsection{Cylindrical tungsten collimator}

The dose enhancement effect of the cylindrical tungsten collimator on the surrounding tissue was evaluated by performing Geant 4 simulations with and without the probe in a water phantom. The collimator was modelled as a $100 \mathrm{~mm}$ long half-cylindrical tungsten shell with an inner radius of $8 \mathrm{~mm}$ and an outer radius of $12 \mathrm{~mm}$. This was placed at the centre of a $300 \times 300 \times 300 \mathrm{~mm}$ cubic water phantom. The simulation model is illustrated in figure 3 . The sensitive volume used to evaluate the dose was constructed by quantising the volume of water surrounding the probe into scoring voxels with a cylindrical coordinate system. The sensitive 
volume extends $41 \mathrm{~mm}$ along the axis of the collimator $(y \in\{-20,-19, \cdot, 19,20\} \mathrm{mm})$, divided into $411 \mathrm{~mm}$ slices; from $12 \mathrm{~mm}$ to $22 \mathrm{~mm}$ radially (along the $r$ axis), divided into 101 concentric annular rings $(r \in\{12.05,12.15, \cdot, 21.95,22.05\} \mathrm{mm})$, and spans $170^{\circ}$ in the azimuth divided into $1710^{\circ}$ sectors $\left(\phi \in\left\{-80^{\circ},-70^{\circ}, \cdot,+70^{\circ},+80^{\circ}\right\}\right)$. Therefore, the dose is calculated for $41 \times 17 \times 101=70397$ voxels, where each voxel is addressed by its displacement along the collimator's axis $(y)$, the azimuth of its sector $(\phi)$ and its radial distance from the probe axis $(r)$. Again, BSDF is calculated as the ratio of the dose measured in each scoring voxel with and without the collimator present.

\section{Results}

The following sections detail the simulation results for both collimators, together with experimental validation of the results for the planar collimator. All uncertainties quoted or illustrated on graphs correspond to three standard deviations $(k=3,99 \%$ confidence intervals, where only Type A uncertainties are taken into account). $d_{\text {SC }}$ denotes the distance between the source and the centre of the top surface of the planar collimator or the highest point of the cylindrical collimator; $x$ denotes the distance from the top surface of the collimator to the point at which dose is measured.

\subsection{Planar tungsten collimator}

3.1.1. Monte Carlo simulations. The BSDF values calculated for the two simulated source positions with and without the planar tungsten collimator (described in section 2.1.1) are plotted as a function of distance $x$ from the top surface of the collimator in figure 4 . For both source positions, the BSDF approaches unity beyond a distance of $0.5 \mathrm{~mm}$ from the collimator. The largest BSDF occurs near the surface of the tungsten collimator. As shown in figures $4(a)$ and $(b)$, the average BSDFs within $0.1 \mathrm{~mm}$ are $2.55 \pm 0.22$ and $2.68 \pm 0.27$ recorded for the source placed at $20 \mathrm{~mm}$ and $43 \mathrm{~mm}$ above the top surface of the tungsten collimator, respectively.

The BSDF is additionally evaluated at a higher spatial resolution (using $10 \mu \mathrm{m}$ thick scoring voxels) within the first $500 \mu \mathrm{m}$ of the surface of the planar tungsten collimator; results are shown in figures $4(c)$ and $(d)$. A larger maximum BSDF and steeper BSDF gradient is observed when the source is placed $43 \mathrm{~mm}$ above the top surface of the collimator. The BSDFs in the sensitive volume $70 \mu \mathrm{m}$ above the collimator (the water equivalent depth of the MOSkin sensor) are $1.81 \pm 0.40$ and $1.91 \pm 0.31$ for the source placed at $20 \mathrm{~mm}$ and $43 \mathrm{~mm}$ above the collimator, respectively.

3.1.2. Experimental validation. The BSDFs experimentally measured using the MOSkin detector are shown in table 1.

The results agree within uncertainties for both source positions. The increase of dose due to the presence of the tungsten plate is only observed in the measurement where the MOSkin is flush with the top surface of tungsten collimator.

\subsection{Cylindrical tungsten collimator}

Figure 5 shows the BSDF as a function of distance from the top of the cylindrical collimator $\left(\phi=0^{\circ}, y=0 \mathrm{~mm}\right)$. As distance from the cylindrical collimator increases, the behaviour of the BSDF is similar to that exhibited in the simulation of the planar tungsten collimator. 


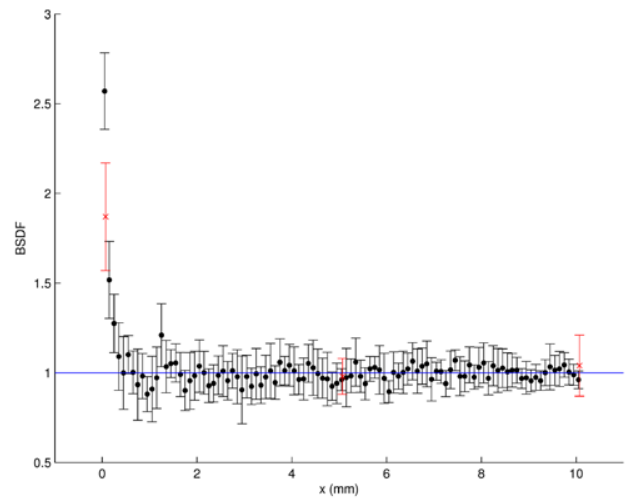

(a)

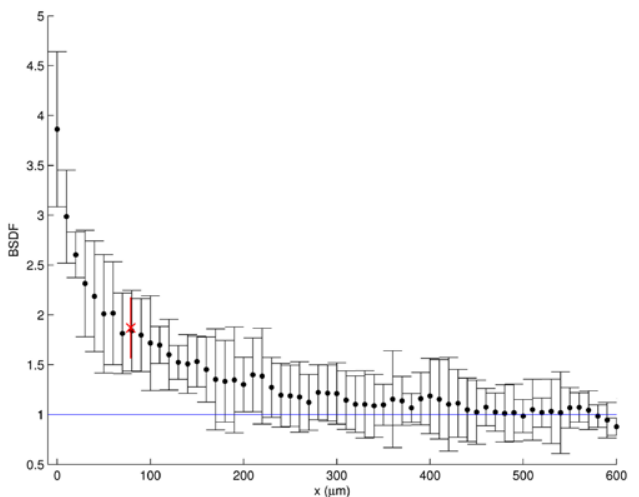

(c)

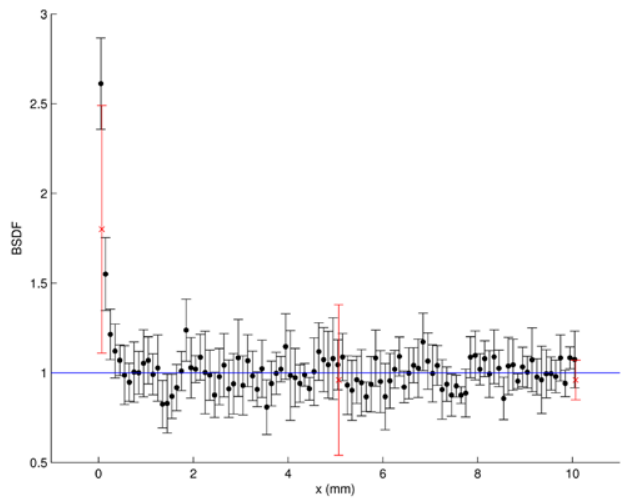

(b)

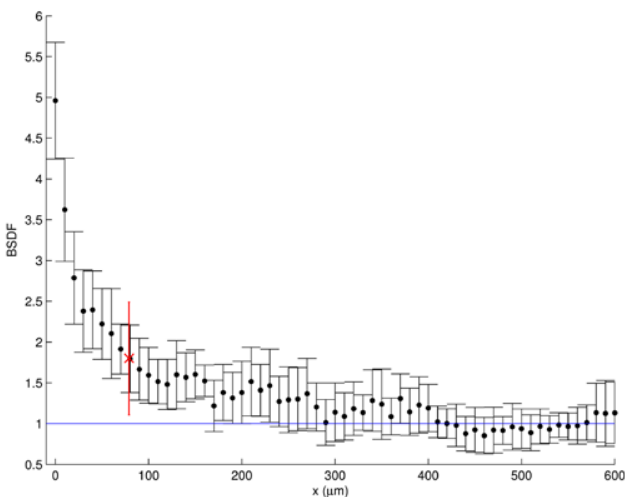

(d)

Figure 4. Simulated backscatter dose fraction (BSDF) as a function of distance for the source positioned at two distances above a $4 \mathrm{~mm}$ thick planar tungsten collimator. For comparison, experimentally-measured values sampled at a depth of $70 \mu \mathrm{m}$ are shown in red. The calculated BSDFs approach a value of 1 beyond a distance of $0.5 \mathrm{~mm}$ from the top surface of the tungsten collimator. $99 \%$ confidence intervals are shown. (a) $\phi=0^{\circ}$, $y=0 \mathrm{~mm} d_{\mathrm{SC}}=20 \mathrm{~mm}$. (b) $\phi=0^{\circ}, y=0 \mathrm{~mm} d_{\mathrm{SC}}=43 \mathrm{~mm}$. (a) $\phi=0^{\circ}, y=0 \mathrm{~mm}$ $d_{\mathrm{SC}}=20 \mathrm{~mm}$. (a) $\phi=0^{\circ}, y=0 \mathrm{~mm} d_{\mathrm{SC}}=43 \mathrm{~mm}$.

Table 1. BSDF measured using MOSkin detector. Associated uncertainties are $k=3$.

\begin{tabular}{ccccc}
\hline & \multirow{2}{*}{$\begin{array}{c}\text { Detector face } \\
\text { depth }(\mathrm{mm})\end{array}$} & $\begin{array}{l}\text { Sensitive } \\
\text { depth }(\mathrm{mm})\end{array}$ & $d_{\mathrm{SC}}=20 \mathrm{~mm}$ & $d_{\mathrm{SC}}=43 \mathrm{~mm}$ \\
\cline { 3 - 4 } & 0 & 0.07 & $1.87 \pm 0.30$ & $1.80 \pm 0.69$ \\
& 5 & 5.07 & $0.98 \pm 0.10$ & $0.96 \pm 0.42$ \\
& 10.07 & $1.04 \pm 0.17$ & $0.96 \pm 0.11$ \\
\hline
\end{tabular}

Figures $6(c)$ and $(d)$ show that the BSDF around the tungsten collimator is essentially independent of axial displacement for all values of $r$.

Figure 6(a) indicates that when the source is $20 \mathrm{~mm}$ from the collimator, the BSDF is almost independent of $|\phi|$ when $|\phi| \leqslant 60^{\circ}$ with a peak value of 2.4 , falling sharply to unity 


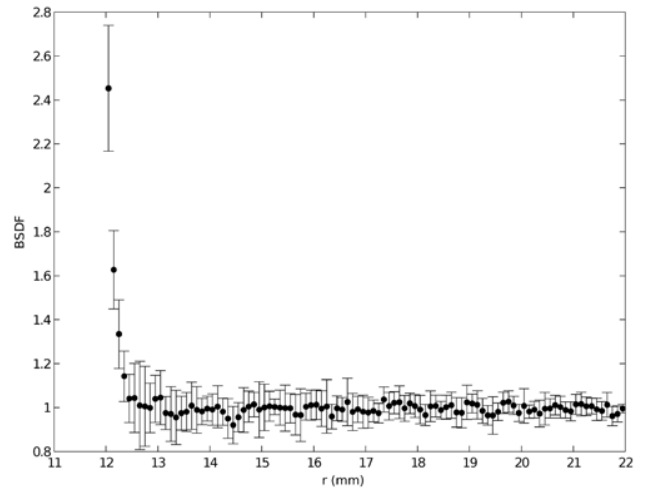

(a)

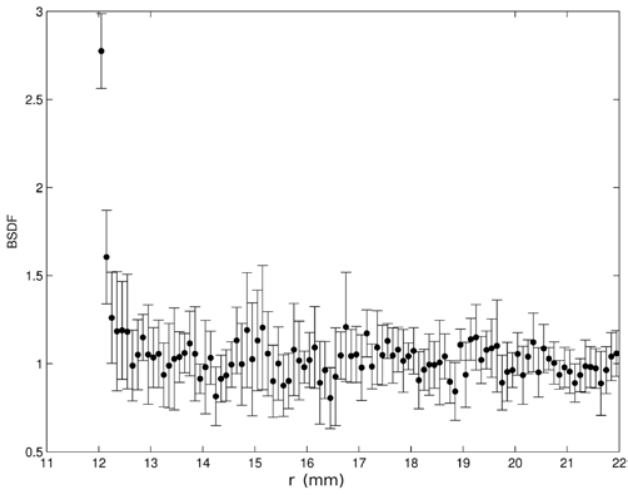

(b)

Figure 5. BSDF directly above the centre of the simulated cylindrical tungsten collimator as a function of $r\left(y=0 \mathrm{~mm}\right.$ and $\left.\phi=0^{\circ}\right)$ for two source positions. 99\% confidence intervals are shown. (a) $\phi=0^{\circ}, y=0 \mathrm{~mm} d_{\mathrm{SC}}=20 \mathrm{~mm}$. (b) $\phi=0^{\circ}, y=0 \mathrm{~mm}$ $d_{\mathrm{SC}}=43 \mathrm{~mm}$

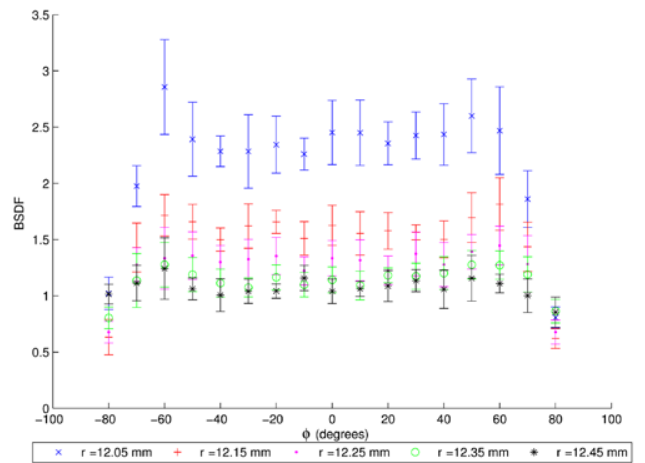

(a)

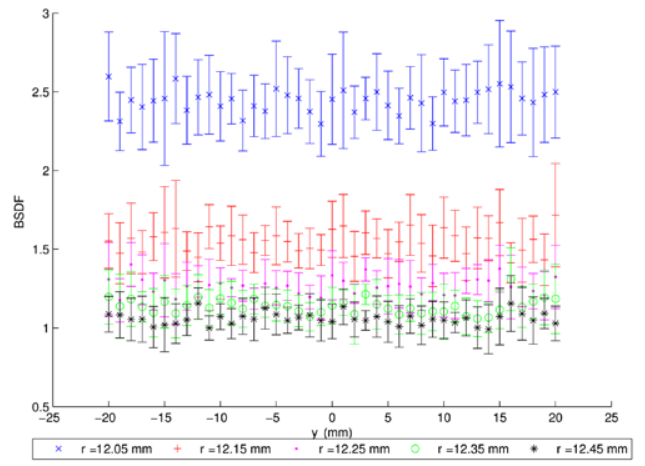

(c)

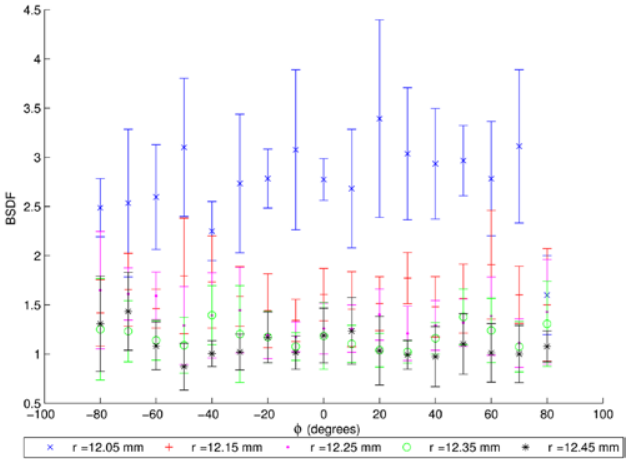

(b)

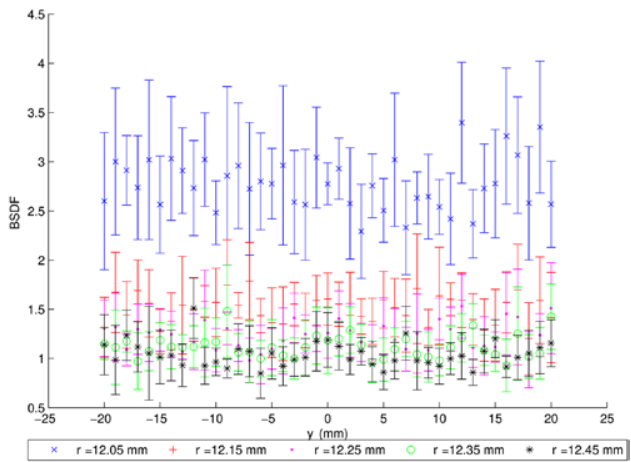

(d)

Figure 6. BSDF from the simulated cylindrical tungsten collimator as a function of $\phi$ and $y$, calculated for four different values of $r$ and two source positions $\left(d_{\mathrm{SC}}=43 \mathrm{~mm}\right.$ and $d_{\mathrm{SC}}=20 \mathrm{~mm}$ ). (a) $y=0 \mathrm{~mm} d_{\mathrm{SC}}=20 \mathrm{~mm}$. (b) $y=0 \mathrm{~mm} d_{\mathrm{SC}}=43 \mathrm{~mm}$. (c) $\phi=0^{\circ}$, $d_{\mathrm{SC}}=20 \mathrm{~mm} .(d) \phi=0^{\circ}, d_{\mathrm{SC}}=43 \mathrm{~mm}$. 


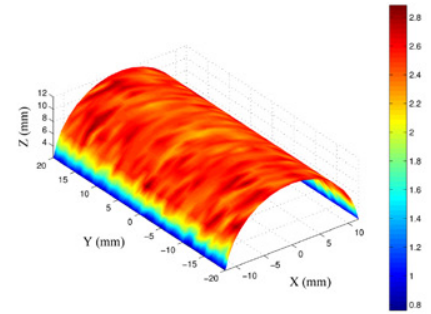

(a)

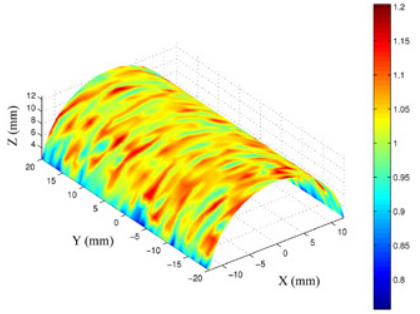

(b)

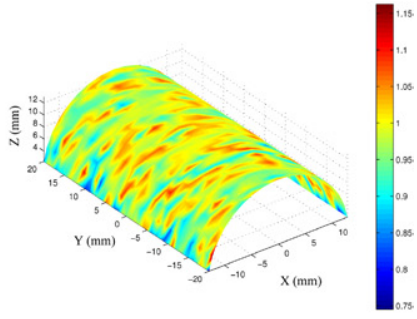

(c)

Figure 7. BSDF values from the simulated cylindrical tungsten collimator as a function of $y$ and $\phi$ were computed for three values of $r$ and are shown as heatmap surface plots in three dimensions, for the source placed at $20 \mathrm{~mm}$ above the collimator $\left(d_{\mathrm{SC}}=20 \mathrm{~mm}\right)$. $99 \%$ confidence intervals are shown. (a) $r=12.05 \mathrm{~mm}$. (b) $r=12.55 \mathrm{~mm}$. (c) $r=13.05 \mathrm{~mm}$.

when $60^{\circ}<|\phi|<80^{\circ}$ for a radial distance of $12.05 \mathrm{~mm}$. For larger values of $r$, the fall-offs in BSDF still occur at a similar angular threshold but are less pronounced: while the maximum BSDF is 2.4 for $r=12.05 \mathrm{~mm}$, the BSDF remains essentially constant with respect to $\phi$ for all values of $r$ and is close to unity for $r=12.45 \mathrm{~mm}$. A similar trend is observed in the plotted BSDF values in figure $6(b)$, where the source is placed $43 \mathrm{~mm}$ above the cylindrical collimator, demonstrating a sharp reduction in BSDF with increasing radial distance.

Heatmap surfaces illustrating the BSDF as a function of $y$ and $\phi$ for $r=12.05 \mathrm{~mm}$, $12.55 \mathrm{~mm}$ and $13.05 \mathrm{~mm}$ are shown in figure 7. For each value of $r$, the BSDF remains nearly constant with respect to the $y$ axis, and its minimum value occurs when $|\phi|>80^{\circ}$.

\section{Discussion}

The simulations indicate that although there is significant dose enhancement in the vicinity of the tungsten-phantom interface, the BSDF rapidly declines to unity (that is, no measurable backscatter dose enhancement) within a distance of less than $1 \mathrm{~mm}$ from the collimator surface, both for planar and cylindrical tungsten collimators. For the planar collimator, this result has been experimentally confirmed; at the closest practical point of measurement (equivalent to approximately $70 \mu \mathrm{m}$ from the collimator surface) the BSDF was measured to be $1.87 \pm 0.30$ and $1.80 \pm 0.69$ for a source-collimator distance of $20 \mathrm{~mm}$ and $43 \mathrm{~mm}$, respectively, while the dose measurements acquired at distances of $5 \mathrm{~mm}$ and $10 \mathrm{~mm}$ from the surface of the collimator show no measurable dose enhancement (BSDF is very close to unity). Therefore, based on the MOSkin measurements it is safe to conclude that dose enhancement is insignificant $5 \mathrm{~mm}$ from the collimator surface in a tissue-equivalent medium, and according to the Monte Carlo simulations, is expected to be negligible beyond $1 \mathrm{~mm}$ from the collimator surface. In the complete BrachyView probe design, the collimator is encased in a protective $1 \mathrm{~mm}$ thick medical-grade sterilisable tissue-equivalent plastic shell—therefore the vast majority of the backscatter dose will be absorbed in this shell.

The results of the experiments agreed well with the high-resolution simulation conducted using a scoring volume with a thickness of $10 \mu \mathrm{m}$. Since the MOSkin detector has a WED of $70 \mu \mathrm{m}$, the measurements taken using MOSkin when placed flush with the collimator are effectively performed at a distance equivalent to $70 \mu \mathrm{m}$ above the tungsten plate, within a sensitive volume less than $1 \mu \mathrm{m}$ thick. The experimental results are in good agreement with the 
value obtained in simulations when the sensitive volume is placed $70 \mu \mathrm{m}$ above the top surface of the collimator. The results obtained demonstrate the ability of the MOSkin dosimeter to accurately characterise a very steep sub-millimetre dose gradient.

Although the dose measured at the tungsten interface is more than double the dose measured in the absence of the tungsten plate, this dose increase is limited to a very short distance (less than $1 \mathrm{~mm}$ ) from the interface between the tungsten collimator and the water-equivalent phantom. This is because the backscatter dose is mostly contributed by low energy secondary electrons (Das and Chopra 1995). This result agrees with the behaviour described in studies published in 2004 and 2006 by Ravikumar et al and Sathiyan et al respectively (Ravikumar et al 2004, Sathiyan et al 2006), which showed that lower incident photon energy will lead to a shorter backscatter electron range. The lowest energy included in these studies is $1.17 \mathrm{MeV}$ $\left({ }^{60} \mathrm{Co}\right)$ and a backscatter dose enhancement range of a few millimetres was obtained. Since the average energy of the encapsulated ${ }^{192} \mathrm{Ir}$ is around $360 \mathrm{keV}$ (Ash et al 2002) with a primary emission spectrum that extends to approximately $612 \mathrm{keV}$ (Rivard et al 2010) and an additional component of secondary scattered photons which accumulate in the low-energy peak around $60 \mathrm{keV}$ (Anagnostopoulos et al 2003), the range of backscatter electrons is expected to be very short.

In ${ }^{192}$ Ir HDR brachytherapy, the average photon energy decreases as the depth of tissue penetration increases, with an increase in the fluence of low-energy scattered photons (Zilio et al 2006). Therefore, as the distance from the source to the tungsten-tissue interface increases from $20 \mathrm{~mm}$ to $43 \mathrm{~mm}$ in the prostate volume, the rate of backscattered electron emission actually increases, although the mean electron range is shorter due to the larger proportion of lower energy incident photons. This hypothesis is confirmed in our simulations, which show that although the BSDF is higher immediately adjacent to the tungsten collimator, it decreases more rapidly with distance from the collimator for $d_{\mathrm{SC}}=43 \mathrm{~mm}$ compared to $d_{\mathrm{SC}}=20 \mathrm{~mm}$, for both planar and cylindrical collimators.

Simulations of the cylindrical tungsten collimator show that the BSDF is approximately independent of displacement along the axis of the collimator (for $\phi=0^{\circ}$ and constant $r$ ), which is in agreement with previously-published results (Ravikumar et al 2004). The decrease in the BSDF at large values of $\phi$ (when $y=0 \mathrm{~mm}$ and $r$ is very small $(12.05 \mathrm{~mm}$ and $12.15 \mathrm{~mm}$ )) for $d_{\mathrm{SC}}=20 \mathrm{~mm}$ occurs because at these angles, the tungsten-tissue interface is not directly illuminated by the source and is instead partially shielded by the adjacent wall of the collimator, reducing the direct dose received. This reduction is less pronounced at $d_{\mathrm{SC}}=43 \mathrm{~mm}$, since due to the geometry of the situation, photons must pass through a smaller section of the collimator wall before reaching the tissue-tungsten interface at extreme values of $\phi$. Additionally, compared to the $d_{\mathrm{SC}}=20 \mathrm{~mm}$ case, at $d_{\mathrm{SC}}=43 \mathrm{~mm}$, a larger proportion of the photons arriving at the interface have been scattered, and have not passed through the collimator at all since they are distributed almost isotropically at low energies (Das and Chopra 1995). This increases the BSDF at large values of $\phi$ relative to the $d_{\mathrm{SC}}=20 \mathrm{~mm}$ case.

\section{Conclusion}

Experimental and simulation studies have been performed to investigate the backscatter dose introduced by the tungsten collimator in the HDR BrachyView project. Both Monte Carlo simulations and experiments were performed in the case of a planar tungsten collimator, while a cylindrical collimator was investigated through simulation only. Good agreement has been obtained between experiment and simulations, confirming that the patient's anterior rectal wall will not be exposed to a measurable dose increase in the vicinity of the collimator when 
used in the final BrachyView design. This is because even though the dose is significantly enhanced at the surface of the collimator, the short range of the backscattered recoil electrons is such that all backscatter dose is deposited within $1 \mathrm{~mm}$ of the collimator surface. Since this region is occupied by a tissue-equivalent plastic shell, the patient will not receive any additional dose in the rectal wall or other tissues, confirming that the use of BrachyView will not cause additional radiation injury to the patient, while providing real-time in vivo QA of treatment delivery.

\section{Acknowledgments}

Financial support for the research and development of the BrachyView probe was provided by the National Health and Medical Research Council (NHMRC) research grant 573428.

\section{References}

Anagnostopoulos G, Baltas D, Geretschlaeger A, Martin T, Papagiannis P, Tselis N and Zamboglou N 2003 In vivo thermoluminescence dosimetry dose verification of transperineal 192Ir high-dose-rate brachytherapy using CT-based planning for the treatment of prostate cancer Int. J. Radiat. Oncol. 57 1183-91

Ash D et al 2002 The GEC ESTRO handbook of brachytherapy Eur. Soc. Therapeutic Radiol. Oncol. 25

Batic M, Burger J, Cindro V, Kramberger G, Mandic I, Mikuz M, Studen A and Zavrtanik M 2011 Verification of high dose rate Ir-192 source position during brachytherapy treatment using silicon pixel detectors IEEE Trans. Nucl. Sci. 58 2250-6

Beaulieu L, Tedgren A, Carrier J F, Davis S D, Mourtada F, Rivard M J, Thomson R M, Verhaegen F, Wareing T A and Williamson J F 2012 Report of the task group 186 on model-based dose calculation methods in brachytherapy beyond the TG-43 formalism: current status and recommendations for clinical implementation Med. Phys. 39 6208-36

Borg J and Rogers D W O 1998 Monte Carlo calculations of photon spectra in air from 192 Ir sources NRC Report PIRS-629r

Castillo M H, Button T M, Doerr R, Homs M I, Pruett C W and Pearce J I 1988 Effects of radiotherapy on mandibular reconstruction plates Am. J. Surg. 156 261-3

Chin D W H, Treister N, Friedland B, Cormack R A, Tishler R B, Makrigiorgos G M and Court L E 2009 Effect of dental restorations and prostheses on radiotherapy dose distribution: a Monte Carlo study J. Appl. Clin. Med. Phys. 10 80-9

Cullen D E, Hubbell J H and Kissel L 1997 EPDL97: the Evaluated Photon Data Library, '97 Version Technical Report UCRL-50400, vol 6, Rev 5, Lawrence Livermore National Laboratory Livermore, CA, USA

Cunningham J, Coffey M, Knöös T and Holmberg O 2010 Radiation oncology safety information system (ROSIS) — profiles of participants and the first 1074 incident reports Radiother. Oncol. 97 601-7

Daskalov G M, Löffler E and Williamson J F 1998 Monte Carlo-aided dosimetry of a new high dose-rate brachytherapy source Med. Phys. 25 2200-8

Das I J and Chopra L K 1995 Backscatter dose perturbation in kilovoltage photon beams at high atomic number interfaces Med. Phys. 22 767-73

Das I J and Kahn F M 1989 Backscatter dose perturbation at high atomic number interfaces in megavoltage photon beams Med. Phys. 16 367-75

Farahani M, Eichmiller F C and McLaughlin W L 1990 Measurement of absorbed doses near metal and dental material interfaces irradiated by $\mathrm{x}-$ and gamma-ray therapy beams Phys. Med. Biol. 35 369-85

Kertzscher G, Andersen C E, Siebert F A, Nielsen S K, Lindegaard J C and Tanderup K 2011 Identifying afterloading $\{\mathrm{PDR}\}$ and $\{\mathrm{HDR}\}$ brachytherapy errors using real-time fiber-coupled Al2O3:C dosimetry and a novel statistical error decision criterion Radiother. Oncol. 100 456-62

Kwan I S et al 2008 Skin dosimetry with new MOSFET detectors Radiat. Meas. 43 929-32 
Kwan I S, Wilkinson D, Cutajar D, Lerch M, Rosenfeld A, Howie A, Bucci J, Chin Y and Perevertaylo V L 2009 The effect of rectal heterogeneity on wall dose in high dose rate brachytherapy Med. Phys. 36 224-32

Nadrowitz R and Feyerabend T 2001 Backscatter dose from metallic materials due to obliquely incident high-energy photon beams Med. Phys. 28 959-65

Nath R, Anderson L L, Luxton G, Weaver K A, Williamson J F and Meigooni A S 1995 Dosimetry of interstitial brachytherapy sources: recommendations of the AAPM radiation therapy committee task group No. 43 Med. Phys. 22 209-34

Niroomand-Rad A, Razavi R, Thobejane S and Harter K W 1996 Radiation dose pertubation at tissuetitanium dental interfaces in head and neck cancer patients Int. J. Radiat. Oncol. 34 475-80

Perez-Calatayud J, Ballester F, Das R K, DeWerd L A, Ibbott G S, Meigooni A S, Ouhib Z, Rivard M J, Sloboda R S and Williamson J F 2012 Dose calculation for photon-emitting brachytherapy sources with average energy higher than $50 \mathrm{keV}$ : report of the AAPM and ESTRO Med. Phys. 39 2904-29

Qi Z, Deng X, Huang S, Lu J, Lerch M, Cutajar D and Rosenfeld A 2007 Verification of the plan dosimetry for high dose rate brachytherapy using metal-oxide-semiconductor field effect transistor detectors Med. Phys. 34 2007-13

Ravikumar M, Ravichandran R, Sathiyan S and Supe S S 2004 Backscattered dose perturbation effects at metallic interfaces irradiated by high-energy x- and gamma-ray therapeutic beams Strahlenther. Onkol. 108 173-8

Reft C, Alecu R, Das I J, Gerbi B J, Keall P, Lief E, Mijnheer B J, Papanikolaou N, Sibata C and Dyk J V 2003 Dosimetric considerations for patients with HIP prostheses undergoing pelvic irradiation. Report of the AAPM radiation therapy committee task group 63 Med. Phys. 30 1162-82

Regulla D F, Hieber L B and Seidenbusch M 1998 Physical and biological interface dose effects in tissue due to x-ray-induced release of secondary radiation from metallic gold surfaces Radiat. Res. $15092-100$

Rivard M J, Coursey B M, DeWerd L A, Hanson W F, Huq M S, Ibbott G S, Mitch M G, Nath R and Williamson J F 2004 Update of AAPM Task Group No. 43 Report: a revised AAPM protocol for brachytherapy dose calculations Med. Phys. 31 633-74

Rivard M J, Granero D, Perez-Calatayud J and Ballester F 2010 Influence of photon energy spectra from brachytherapy sources on Monte Carlo simulations of kerma and dose rates in water and air Med. Phys. 37 869-76

Rivard M J, Venselaar J L M and Beaulieu L 2009 The evolution of brachytherapy treatment planning Med. Phys. 36 2136-53

Safavi-Naeini M et al 2013 BrachyView, a novel inbody imaging system for HDR prostate brachytherapy: design and Monte Carlo feasibility study Med. Phys. 40071715

Sathiyan S, Ravikumar M and Supe S S 2006 Measurement of backscattered dose at metallic interfaces using high energy electron beams Rep. Pract. Oncol. Radiother. 11 117-21

Thomadsen B, Lin S W, Laemmrich P, Waller T, Cheng A, Caldwell B, Rankin R and Stitt J 2003 Analysis of treatment delivery errors in brachytherapy using formal risk analysis techniques Int. $J$. Radiat. Oncol. 57 1492-508

Valentin J 2005 Prevention of high-dose-rate brachytherapy accidents. ICRP publication 97 Ann. ICRP $351-51$

Williamson J F 2006 Brachytherapy technology and physics practice since 1950: a half-century of progress Phys. Med. Biol. 51 R303-25

Zilio V O, Joneja O P, Popowski Y, Rosenfeld A and Chawla R 2006 Absolute depth-dose-rate measurements for an Ir192 HDR brachytherapy source in water using MOSFET detectors Med. Phys. 33 1532-9 